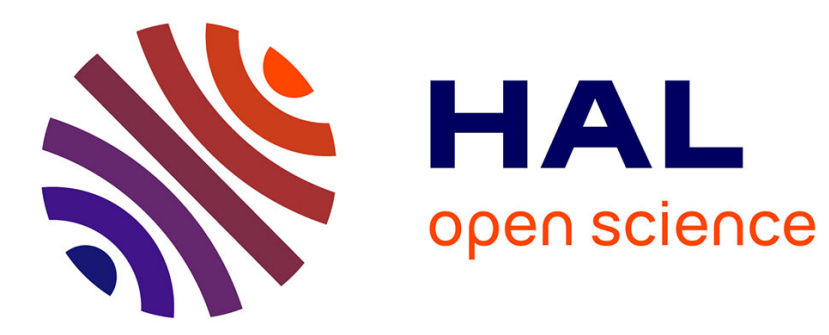

\title{
Planning in-hand dexterous micro-manipulation using 3-D rotations decomposition
}

\author{
Pardeep Kumar, Redwan Dahmouche, Michaël Gauthier
}

\section{To cite this version:}

Pardeep Kumar, Redwan Dahmouche, Michaël Gauthier. Planning in-hand dexterous micromanipulation using 3-D rotations decomposition. 17th International Conference on Automation Science and Engineering (CASE), Aug 2021, Lyon, France. hal-03455030

\section{HAL Id: hal-03455030 \\ https://hal.science/hal-03455030}

Submitted on 29 Nov 2021

HAL is a multi-disciplinary open access archive for the deposit and dissemination of scientific research documents, whether they are published or not. The documents may come from teaching and research institutions in France or abroad, or from public or private research centers.
L'archive ouverte pluridisciplinaire HAL, est destinée au dépôt et à la diffusion de documents scientifiques de niveau recherche, publiés ou non, émanant des établissements d'enseignement et de recherche français ou étrangers, des laboratoires publics ou privés. 


\title{
Planning in-hand dexterous micro-manipulation using 3-D rotations decomposition
}

\author{
Pardeep KUMAR, Redwan DAHMOUCHE, Michaël GAUTHIER
}

\begin{abstract}
This paper aims to contribute to the improvement of dexterity in contact micro-manipulation by performing inhand dexterous micro-manipulation planning. Previous experimental works on planar micro-manipulation showed that such an approach allows for large rotations of arbitrary shaped objects. Moving from planar to 3-D manipulation significantly increases the complexity of the manipulation planning, especially when considering the rolling of the fingers on the object during the manipulation. We propose in this paper a dexterous manipulation planning algorithm that leverages the complexity of 3-D manipulation planning by decomposing the desired 3-D rotations into three successive rotations within two different planes. Optimal paths of the manipulating fingers are thus obtained in the planar spaces and then combined to form the trajectories in the 3-D space. Besides the relevance of the approach, the simulation results show that exploiting adhesion forces improves the robustness of the manipulation and extends the manipulation capabilities, but at the expense of the computation time.
\end{abstract}

Index Terms-Micro-manipulation, Manipulation Planning, Dexterity, Grasping, In-hand manipulation.

\section{INTRODUCTION}

Dexterous manipulation at the macro-scale has been an active research topic in the last three decades [1]-[3]. Different operations like rolling [4], sliding [5], and finger gaiting [6] have been studied to perform in-hand manipulation tasks [7]. The demonstrated capabilities range from simple pick-andplace to more complex tasks, such as in-hand rotation of a cube [8], picking up a coin from the floor [9], opening a bottle cap [10], and many others.

At the micro-scale, manipulation methods are classified into two groups: (i) non-contact manipulation, in which the object trajectory is controlled with a physical field (e.g. electric, magnetic, etc.) and; (ii) contact-based manipulation, where the manipulator is in physical contact with manipulated object, just like the way humans manipulate common objects. This paper focuses on this second category, which is currently limited to basic tasks [11]-[15].

In contact based micro-manipulations, two main types of robot architectures are proposed. The first one consists of a basic tweezers mounted on a precision manipulation robot. Yet, we face hurdles in achieving the desired rotations using this architecture, while achieving translations is usually more trivial. Indeed, the uncertainties and variations of the robot

FEMTO-ST Institute, Univ. Bourgogne Franche-Comté CNRS, Besançon, France. (firstname. lastnamed femto-st.fr) kinematic parameters and the existence of mechanical defects (backlash, out of roundness, etc.) affect the position of the rotation center of the tweezers and introduce undesired motion of the manipulated object [16]. In addition, the entire robot has to rotate in order to manipulate the micro-object, which requires a large space. Unfortunately, such spaces are not available in many applications such as in Scanning Electron Microscope's chambers or in several medical applications. Such constraints limit the feasibility of this approach.

The second approach in contact micro-manipulation is to use two or more decoupled probes or fingers that have several Degrees of Freedom (DoF). In this approach, the trajectory planning of the fingers is more complex but the manipulation of the objects requires less space since it is done in the manipulating "micro-hand" [17], [18]. However, most of the works that use this approach are limited to simple object shapes like spheres [19]-[21]. Other works consider more complex shapes but the dexterity of the proposed approaches is limited. For instance, two fingers are used in [22] to rotate a Lego-like object but the rotation angles are small since no re-grasp operation is performed. In [23], the manipulated object lays on a substrate (2-D motion) and is pushed by four probes to get to the desired object's pose. The manipulation is thus limited to a horizontal plane.

Contact micro-manipulation is also made difficult because of surface forces (capillary, van der Waals and electrostatic). Those forces are often dominant over gravitational and inertial forces which make the manipulated objects stick to substrate and the tweezers [24]. For a long time, adhesion forces were seen as disturbing effects that we have to mitigate [25]. However, it was recently shown that such forces can be exploited to improve dexterous micromanipulation capabilities. Indeed, the adhesion forces allow the fingers to pull the object which enhances the stability of the grasps [26]. In [27], Seon et al. demonstrated that in-hand planar micro-manipulation of arbitrary shaped planar objects can be achieved in presence of adhesion forces. Dexterous manipulations with rotations above $220^{\circ}$ for a wide variety of planar objects at the micro-scale were experimentally achieved.

The aim of this work is to enhance the capabilities of manipulating micro-objects with complex shapes in the 3D space. The main challenge in performing 3-D dexterous manipulation in general, and micro-manipulation in particular, is the complexity of the manipulation planning algorithm 
that might increase exponentially with the dimension of the configuration space of the object and that of the hand.

In this article, we develop a dexterous 3-D manipulation algorithm with a moderate complexity. The main idea is to perform 3-D dexterous manipulation by using 2-D planners. The proposed approach consists in: i) decomposing 3-D rotations into three individual 2-D rotations; ii) planning the manipulation within the sub-spaces and; iii) combining them to form 3-D manipulation trajectories. The increase in complexity from 2-D manipulation to 3-D is thus moderate (three 2-D paths). The adhesion forces are exploited to stabilize the grasps and the $A^{*}$ search algorithm is used to generate optimal paths in the considered sub-spaces.

\section{MOdeling AND BACKGROUND}

\section{A. Grasping Forces}

To grasp an object with $F$ fingers, the fingers must make contact and apply some grasping force on the object's surface. As proposed in [28], we assume in micro-scale that the contact forces can be modeled using a combination of the Coulomb's Law in which the friction magnitude is independent of the velocity and contact area, and the pull-off force $\left(f_{p o}\right)$ representing the force required to detach the finger from the object as:

$$
\sqrt{f_{x}^{2}+f_{y}^{2}} \leq \mu\left(f_{n}+f_{p o}\right)
$$

where $f_{x}$ and $f_{y}$ are the tangential components of the force on $X$-axis and $Y$-axis respectively, $f_{n}$ is the normal component of force, and $\mu$ is the friction coefficient.

Due to the presence of the pull-off force $f_{p o}$, it is possible to apply a negative force (pulling the object) in micro-scale since the applied force lies in the modified friction cone, whereas in macro-scale only the positive grasping forces (pushing the object) are possible.

\section{B. Grasping Equilibrium}

The manipulated object must be in equilibrium during the whole manipulation process. The equilibrium condition for rigid body is that the sum of all the wrenches should be equal to zero. Whereas, a wrench vector is composed of forces and torques at a contact point as provided by:

$$
w=\left[\begin{array}{l}
f \\
\tau
\end{array}\right]
$$

where $w$ is a wrench, $f$ and $\tau$ represent the force and torque respectively.

Grasp Equilibrium has been discussed in detail in [26], [27], [29]. A grasp using $F$ fingers is stable if:

$$
\sum_{i=1}^{F} w_{i}+w_{e x t}=0,
$$

where $w_{i}$ is the grasping wrench applied by $i^{t h}$ finger, and $w_{\text {ext }}$ is the external wrench applied to the object.

\section{Impact of Pull-off forces on Finger Gaiting}

Pull-off force has an important role in re-grasping and finger gaiting (i.e. fingers replacement). When a finger (e.g. the $F^{\text {th }}$ finger) is being detached, it will pull the object with a force corresponding to the pull-off force $\left(f_{p o}\right)$. This pulloff force may disturb the grasping equilibrium. Thus, it is necessary for the remaining $F-1$ fingers to compensate for this $f_{p o}$ to maintain the object's stability. Thus, removing a finger is possible only if the following equation is satisfied:

$$
\sum_{i=1}^{F-1} w_{i}+w_{\text {ext }}+w_{p o}=0
$$

where $w_{p o}$ is the pull-off wrench caused by releasing the $F^{\text {th }}$ finger.

\section{PRoblem Formalization}

In order to perform fingers path planning, we assume that the object's shape is known through its Computer-Aided Design model (CAD model). Considering the small size of the manipulated object, we also assume that the fingers movement range is significantly larger than the object's dimensions. We also consider only two and three fingers grasps. The admissible contact points on the object that can be used to grasp it are obtained by sampling the object's surface. We then compute the set of all stable grasps, called Maps $(M)$, as well as all the admissible finger gaiting configurations (3 to 2 fingers grasps that can resist a finger detachment). Therefore, there are three different sets of maps: i) initial grasp for which the object can be detached from the substrate $\left(M_{I G}\right)$; ii) object translation and rotation while the fingers roll on the object without sliding $\left(M_{R G}\right)$; iii) and reconfiguration where the number of fingers used for a grasp changes $\left(M_{R C}\right)$.

To generate finger trajectories, the maps are converted to Graph $(G)$, where each stable grasp is connected to its subsequent stable grasp(s). Each element of graph is considered as a Node $(n)$ which are connected by Edges (e). Finding an optimal finger trajectory to rotate an object consists in finding a path in the Graph $(G)$. This step can be performed using a graph search algorithm. Since, a node is an outcome of links of stable grasp configuration of manipulated object, which is based on contact points, fingers, and object orientation; thus, it is possible to represented a node by four parameters i.e. $n=[i j k l]$ where $i, j$, and $k$ define the indexes of contact points on object of the first, second and third fingers respectively, and $l$ defines the index of angular position of the object.

For finger path planning, we are using $A^{*}$ algorithm whose time complexity is $O\left(b^{d}\right)$ where $d$ is the depth (number of nodes to reach the goal) and $b$ is the branching factor (average number of branches per node). There are two cases for branching factor, one is when two fingers are in use, and the second is when three fingers are used. For first case when two fingers are already in contact with object, the branching factor is sum of operations: clockwise rotation, counter clockwise rotation and addition of third finger over 
the remaining $c-2$ contact points. Whereas for the second case when three fingers are in use, the branching factor is sum of operations: clockwise rotation, counter clockwise rotation, and detaching one of the finger.

\section{A. Reducing the complexity using Euler's angle}

Beside the complexity of search algorithm, the major issue to deal with is the number of grasping possibilities $(g p)$, which is the result of the number of contact points $(c)$ of an object, the numbers of fingers $(F)$ being used to grasp the object, and the possible angular positions/orientations $\left(l_{\theta}\right)$ of object (i.e. one turn of rotation $\left(360^{\circ}\right)$ divided by constant rotational step $\Delta \theta)$.

In planar manipulation, where we consider to manipulate an object in a single plane, the number of grasping possibilities is computed as:

$$
g p^{2 D}=c^{F} \cdot l_{\theta},
$$

Whereas, to manipulate an object in spatial case $(\mathcal{S})$, we imagine $\mathcal{P}$ numbers of planes over which the contact points are generated. In this case, the number of grasping possibilities is computed as:

$$
g p^{S D}=(\mathcal{P} \cdot c)^{F} \cdot l_{\theta}=\mathcal{P}^{F} \cdot g p^{2 D}
$$

This increase in the number of grasping possibilities, exponentially increases the complexity of search algorithm.

One of the challenges in 3-D manipulation is to reduce the number of grasping possibilities and search algorithm complexity. According to Euler's Angles, it is possible to decompose any 3-D rotation into three individual 2-D rotations along any two orthogonal axes [30]. Thus keeping that in mind, we propose to only retain two orthogonal planes $\mathcal{P}_{1}$ at $X Y-$ axis and $\mathcal{P}_{2}$ at $X Z$-axis intersecting the object over lines $\mathcal{L}_{1}$ and $\mathcal{L}_{2}$ as illustrated in Fig. 1. Since, the three individual 2-D rotations will be carried out in two different planes, we consider the two intersecting points $I$ and $J$ of lines $\mathcal{L}_{1}$ and $\mathcal{L}_{2}$ as a common link for these three individual rotations. The three individual rotations will be carried out as: $R\left(z, \theta_{1}\right)$ over $\mathcal{P}_{1}, R\left(y, \theta_{2}\right)$ over $\mathcal{P}_{2}$, and $R\left(z, \theta_{3}\right)$ over $\mathcal{P}_{1}$ respectively as represented in Fig. 2 , which will reduce this number of grasping possibilities to sum of grasping possibilities of each rotation carried out over these two planes as:

$$
g p^{3 D}=2 \cdot g p^{2 D_{L_{1}}}+g p^{2 D_{\mathcal{L}_{2}}} .
$$

The reduction of complexity introduced by two planes also reduces the genericity of our approach. Indeed the considered objects should have a geometry in which both planes $\mathcal{P}_{1}$ and $\mathcal{P}_{2}$ can be defined.

\section{B. Sampling strategy}

As we consider that the object's CAD model is known, we sample the manipulation lines $\mathcal{L}_{1}$ and $\mathcal{L}_{2}$ to generate contact

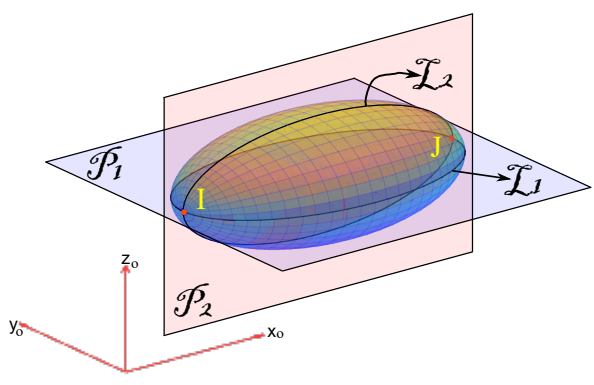

Fig. 1: 3-D object model of ellipsoid and two intersecting orthogonal planes.

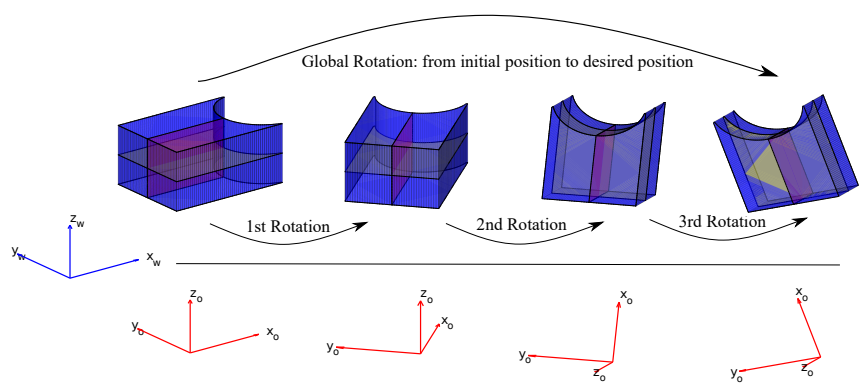

Fig. 2: Decomposition of 3-D rotation in three successive rotations, where blue and red reference frames represent world frame and object frame respectively.

points $(c)$ for grasping. We consider the curvilinear abscissa as the coordinates for each contact point on the object. Whereas, for sampling on the object we considering that the rolling between two successive sample points corresponds to a constant rotation $\Delta \theta$ of the object.

\section{IN-HAND MANipUlation StRATEGY}

Given the number of fingers, and object geometry, the finger trajectory generation is accomplished using two steps. The first is to compute the stable grasps and generate the Graph $(G)$, while the second step is to define a path by traversing the graph(s) to achieve the desired configuration.

Since, the object modeling is restricted only to two planes $\mathcal{P}_{1}$ and $\mathcal{P}_{2}$, and intersecting contact points $I$ and $J$ being the only common link induces some constraints for successive rotations. These constraints are:

- The first rotation should end at the intersecting points I and $J$.

- The second rotation should start from these intersecting points I and J, and end at the same intersecting points.

- The third rotation should start from these intersecting points I and J.

In previous work, Seon et al. [26], [27], [29] achieved only desired pose of object for planar manipulation, while to manipulate the object in 3-D we need to comply with these constraints. Thus, we propose an original method to provide finger trajectories using $A^{*}$ algorithm. 
The $A^{*}$ algorithm uses a heuristic for traversing the graph(s), while ensuring that it computes a path with minimum cost through the nodes $n$. The algorithm optimizes the function $f(n)$, which consists of the cost function $g(n)$ and heuristic function $h(n)$ as:

$$
f(n)=g(n)+h(n) .
$$

\section{A. Cost Function $g(n)$}

Cost function is used to characterize the distance between two nodes. There are three cases for cost function $g(n) ; \mathrm{i})$ initial node cost $g\left(n_{o}\right)$ i.e. when fingers grasp the object for first time, ii) when current node $n$ is reached using finger rolling on object $g(n)^{\text {rolling }}$, iii) when current node $n$ is reached using finger gaiting $g(n)^{\text {gaiting }}$

Initial node cost $g\left(n_{o}\right)$, is the distance between fingers' initial position, and first contact points on object for grasp. When the node $n$ is reached using finger rolling (i.e. without reconfiguration), the cost can be defined as the sum of rolling distance $d_{F_{i}}^{\text {roll }}$ which is the curvilinear abscissa between two contact points covered by each finger $F_{i}$, and previous cost $g\left(n_{\text {previous }}\right)$ as:

$$
g(n)^{\text {rolling }}=\sum_{i=1}^{3} d_{F_{i}}^{\text {roll }}+g\left(n_{\text {previous }}\right) .
$$

Whereas when the node $n$ is reached using a finger reconfiguration (i.e. finger attachment or detachment), the cost $g(n)^{\text {gaiting }}$ will be:

$$
g(n)^{\text {gaiting }}=g_{r}+g\left(n_{\text {previous }}\right),
$$

where $g_{r}$ is a constant and estimated as the minimal distance applied by the actuator to guarantee that the finger is detached. In our case, we choose the value of $g_{r}$ as three times the finger radius.

\section{B. Heuristic Function $h(n)$}

In search algorithms, heuristic function $h(n)$ estimates the minimum cost (in terms of distance for our case) from current node $n$ to the goal node $n_{g}$. As it is important to develop a good heuristic function that guarantees the shortest path; for which the heuristic must underestimates the actual cost. Since, a node is dependent on contact points, fingers being used, and angular position of object, thus we take these parameters into account to formalize our heuristic as:

$$
h(n)=\sum_{i=1}^{3} d_{F_{i}}^{r o l l^{\prime}}+h_{r}(p)
$$

where $d_{F_{i}}^{\text {roll }}$ is the estimated rolling distance covered by finger $F_{i}$ from current node $n$ to goal node $n_{g}$, and $h_{r}$ is the heuristic for reconfiguration, function of parameter $p$ :

$p=\sqrt{\left(i+l-i_{g}-l_{g}\right)^{2}+\left(j+l-j_{g}-l_{g}\right)^{2}+\left(k+l-k_{g}-l_{g}\right)^{2}}$ where current node is $n=\left[\begin{array}{llll}i & j & k & l\end{array}\right]$ and target node is $n_{g}=$ $\left[\begin{array}{llll}i_{g} & j_{g} & k_{g} & l_{g}\end{array}\right]$.

Parameter $p$ checks whether a path between current node $n$ and goal node $n_{g}$ without any reconfiguration exists or not. If such path exists then $p$ will always be zero which will result in $h_{r}(p)$ being zero as well. Otherwise, to reach the goal node $n_{g}$ we will need at least one reconfiguration, and the value of $h_{r}(p)$ in such case will be three times the finger radius.

\section{RESULTS}

The proposed methodology presented in the previous sections, has been simulated and implemented to generate the finger trajectories for three objects with different curvatures in 3-D space i.e. Ellipsoid, Convex shaped object, and Concave shaped object provided in Fig. 3, using three spherical fingers having diameter of $10 \mu \mathrm{m}$ each. For the simulations, we have considered the physical properties of silicon for all fingers and objects, a pull-off force of $\mathbf{1 . 5} \mu N$ [31], and a friction coefficient of $\mathbf{0 . 3}$.

As described in section IV, we propose to decompose the movement in 3 successive rotations as: $R\left(z, \theta_{1}\right)$ over $X Y-$ plane $\mathcal{P}_{1}, R\left(y, \theta_{2}\right)$ over $X Z$ - plane $\mathcal{P}_{2}$, and $R\left(z, \theta_{3}\right)$ over $X Y$ - plane $\mathcal{P}_{1}$ respectively.
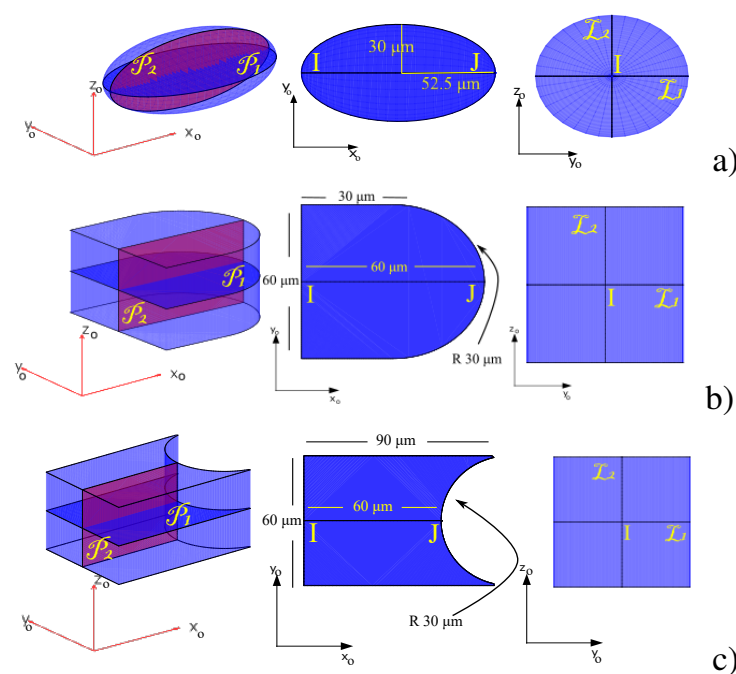

b)

Fig. 3: Different objects a) Ellipsoid, b) Convex Shaped Object, and c) Concave Shaped Object, each with isometric, and 2-D top and side views; used for in-hand dexterous manipulation in 3-D space.

\section{A. Characteristics of the graph $G$}

The Table I represents the total number of nodes for finger path generation against number of contact points over manipulation lines $\mathcal{L}_{1}$ and $\mathcal{L}_{2}$ when adhesion forces are not present. The difference in number of nodes generated is 2 - 4 times higher when adhesion forces are available in comparison to when there is no adhesion force. Indeed, when 
there is adhesion, the object is more stable and a higher number of grasping is stable.

TABLE I: Simulated numerical data for both (i) considering adhesion with a pull-off force of $1.5 \mu N$ and (ii) without adhesion. Finger diameter is $10 \mu m$, friction coefficient $(\mu)$ is 0.3 , and rotational step $(\Delta \theta)$ is $10^{\circ}$.

\begin{tabular}{llcc}
\hline \hline \multirow{2}{*}{ Object } & \begin{tabular}{l} 
Number \\
of contact \\
points \\
\cline { 3 - 4 }
\end{tabular} & \multicolumn{2}{c}{$\begin{array}{c}\text { Number of nodes in the generated } \\
\text { graph (in millions) }\end{array}$} \\
\cline { 3 - 4 } & $\left(\mathcal{L}_{1}, \mathcal{L}_{2}\right)$ & With Adhesion & Without Adhesion \\
\hline Ellipsoid & 362,362 & $337.8,337.8$ & $110.7,110.7$ \\
Convex & 288,302 & $530.1,595$ & $268.8,228.5$ \\
Concave & 440,302 & $1817.4,595$ & $416.2,228.5$ \\
\hline \hline
\end{tabular}

\section{B. Example of finger trajectory}

Fig. 4 - 6 represent an example of the optimal finger path to perform three successive optimal rotations (respectively $40^{\circ}, 70^{\circ}, 20^{\circ}$ ) of Concave Shaped Object. The object is expended on Z-Axis, which is the case for most of the objects at micro-scale built using clean-rooms micro-fabrication methods. We show that the proposed method enables to determine a finger path planning to rotate the micro-object in 3-D space.

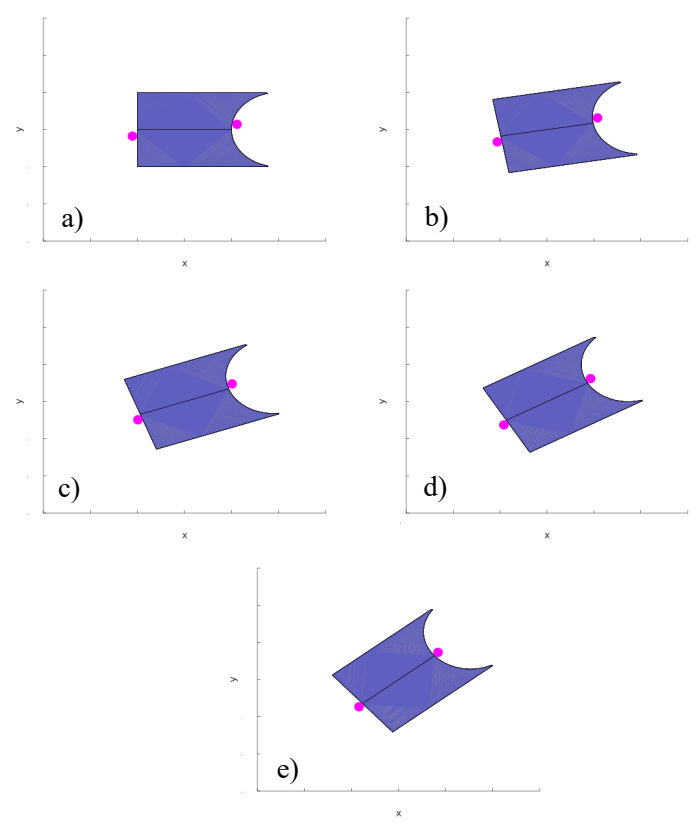

Fig. 4: Concave Shaped Object (2-D top view of $X Y$ plane): Sequence of first rotation of $40^{\circ}$ along XY plane/z-axis; (a)initial grasp of object (b)-(e) rotation of $40^{\circ}$ with rotational step of $10^{\circ}$.

\section{Performance of the path planning}

In order to illustrate the performance of our proposed methods, we determined several optimal finger paths considering the objects presented in Fig. 3. In all the cases, we have considered two possibilities: with adhesion

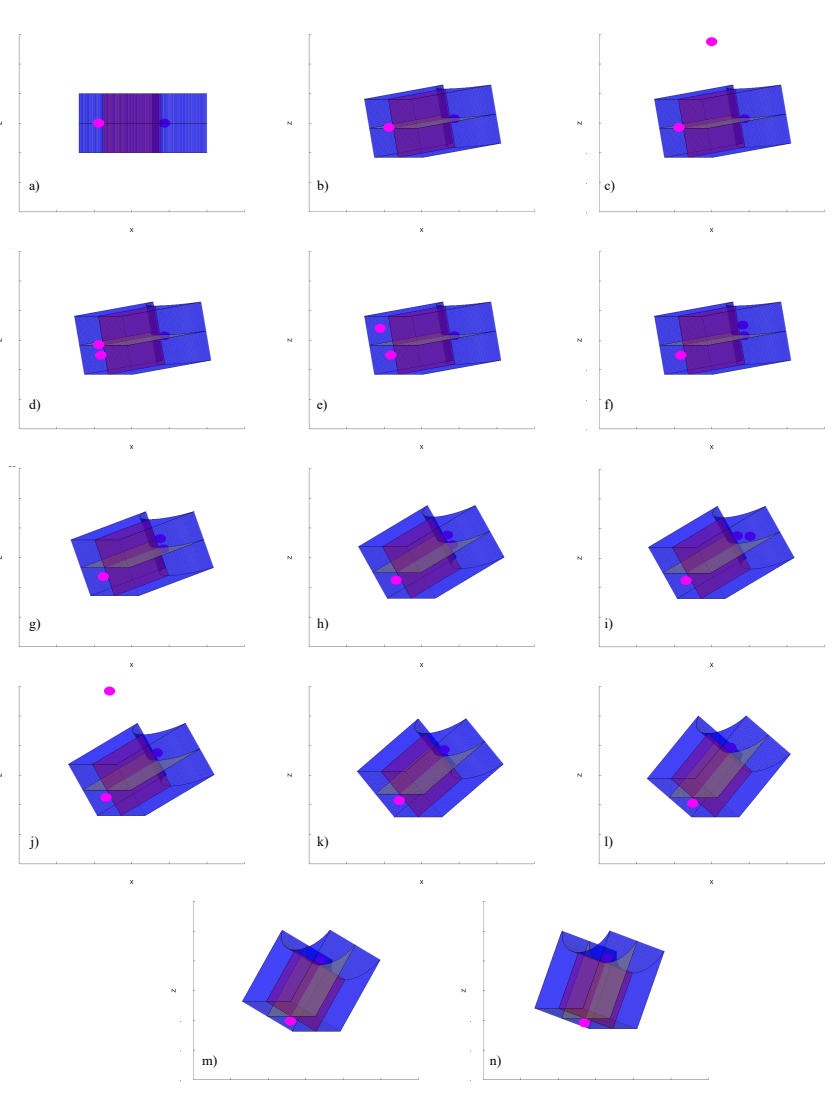

Fig. 5: Concave Shaped Object (2-D side view $X Z$ plane): Sequence of second rotation of $70^{\circ}$ along $X Z$ plane/y-axis; (a) position of fingers on the intersection points $(I, J)$ (sec. III), (b) rotation of rotation of $10^{\circ}$, (c)-(f) reconfiguration of finger composing of finger detachment and finger addition, (g)-(h) rotation of $20^{\circ}$, (i)-(j) finger detachment to go back to its reference position, $(\mathrm{k})-(\mathrm{n})$ rotation of $40^{\circ}$.

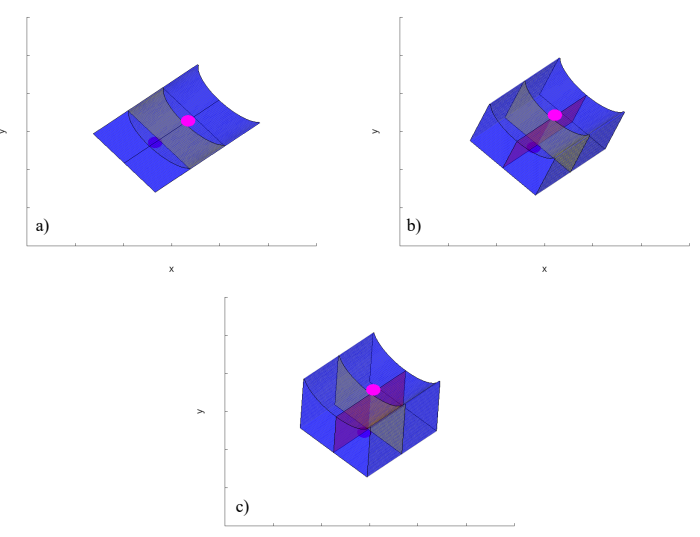

Fig. 6: Concave Shaped Object (2-D top view $X Y$ plane): Sequence of third rotation for $20^{\circ}$ along XY plane/z-axis; (a) last pose from $2^{\text {nd }}$ rotation, (b)-(c) rotation of $20^{\circ}$. 
TABLE II: Comparison of statistical data for an object for both cases with and without the impact of adhesive forces, where W.A., Wo.A.,O.L., C.L., stands for with adhesion, without adhesion, open list (explored by the $\mathrm{A}^{*}$ algorithm) and closed list (describing the optimal path) respectively, and $\theta_{i}$ represents $i^{t h}$ rotation to be carried out for $\theta^{\circ}$.

\begin{tabular}{|c|c|c|c|c|c|c|c|c|c|c|}
\hline \multirow{3}{*}{ Object } & \multirow{2}{*}{\multicolumn{2}{|c|}{ Rotations }} & \multicolumn{4}{|c|}{ Number of nodes in: } & \multirow{2}{*}{\multicolumn{2}{|c|}{ Number of required reconfiguration(s) }} & \multirow{2}{*}{\multicolumn{2}{|c|}{ Time to generate path ( $\mathrm{sec}$ ) }} \\
\hline & & & W. & & & & & & & \\
\hline & $\overline{\theta_{i}}$ & $\theta^{\circ}$ & O.L. & C.L. & O.L. & C.L. & W.A. & Wo.A. & W.A. & Wo.A. \\
\hline \multirow{3}{*}{ Ellipsoid } & $\theta_{1}$ & 40 & 147,612 & 5 & 6,522 & 5 & 0 & 0 & 45 & $<1$ \\
\hline & $\theta_{2}$ & 140 & 2,536 & 19 & 1,689 & 19 & 4 & 4 & 5 & 4 \\
\hline & $\theta_{3}$ & 70 & 946 & 6 & 946 & 6 & 0 & 0 & $<1$ & $<1$ \\
\hline \multirow{3}{*}{ Convex } & $\theta_{1}$ & 80 & 143,824 & 9 & 8,696 & 8 & 0 & 0 & 48 & 1 \\
\hline & $\theta_{2}$ & 90 & 650 & 14 & 930 & 16 & 4 & 6 & 2 & 3 \\
\hline & $\theta_{3}$ & 30 & 682 & 4 & 682 & 4 & 0 & 0 & $<1$ & $<1$ \\
\hline \multirow{3}{*}{ Concave } & $\theta_{1}$ & 40 & 224,458 & 5 & 6,958 & 5 & 0 & 0 & 63 & $<1$ \\
\hline & $\theta_{2}$ & 90 & 428 & 12 & 930 & 16 & 4 & 6 & 2 & 3 \\
\hline & $\theta_{3}$ & 20 & 693 & 3 & 693 & 3 & 0 & 0 & $<1$ & $<1$ \\
\hline
\end{tabular}

and without adhesion to evaluate the impact of adhesion on fingers paths. Various large rotations: for ellipsoid object $\left(40^{\circ}, 30^{\circ}, 50^{\circ}\right),\left(40^{\circ}, 140^{\circ}, 70^{\circ}\right),\left(130^{\circ \dagger}, 150^{\circ}, 90^{\circ}\right)$, $\left(90^{\circ}, 120^{\circ}, 140^{\circ}\right)$, for convex shaped object $\left(40^{\circ}, 70^{\circ \dagger}, 30^{\circ}\right)$, $\left(40^{\circ}, 140^{\circ}, 80^{\circ}\right), \quad\left(90^{\circ}, 160^{\circ}, 30^{\circ}\right), \quad\left(80^{\circ}, 90^{\circ}, 30^{\circ}\right)$, and for concave shaped object $\left(50^{\circ}, 140^{\circ}, 30^{\circ}\right), \quad\left(40^{\circ}, 70^{\circ}, 20^{\circ}\right)$, $\left(40^{\circ}, 90^{\circ}, 20^{\circ}\right),\left(60^{\circ}, 150^{\circ}, 30^{\circ}\right)$ have been carried out. The rotations with $(\dagger)$ represent particular cases where the desired rotation cannot be reached due to the instability of object in the absence of adhesive forces. Indeed, some of the rotations are not accessible without adhesion. It comes from the fact that the graph $\mathrm{G}$ is smaller without adhesion.

The technical data of three simulations are described in Table II. With adhesion forces, the reconfigurations are required only for the $2^{\text {nd }}$ rotations, while in the case of without adhesion forces $\left(60^{\circ}, 150^{\circ}, 30^{\circ}\right.$ for concave shaped object), the other rotations $\left(1^{s t}\right.$ or $3^{r d}$ ) may require reconfiguration(s). The average time to generate: the finger path of $1^{s t}$ rotation for all various object is around 50 seconds (with adhesion) and 1 second (without adhesion), the finger path of $2^{\text {nd }}$ rotation is 3 seconds (with adhesion) and 4 seconds (without adhesion), and the finger path of $3^{r d}$ rotation for all objects is 1 second in both cases with and without adhesion. The time taken to generate the finger path of $1^{s t}$ rotations(s) is higher due to the initial grasp possibilities from fingers' reference position (off-contact) to initial nodes on object (on-contact).

\section{CONCLUSION}

We proposed in this paper a new method to perform finger path planning for 3-D dexterous manipulation of microobjects. To leverage the complexity of 3-D dexterous micromanipulation, we proposed to perform three planar rotations about two perpendicular axes. To ensure the continuity of the manipulation process over the three rotations, we proposed an algorithm that is able to rotate the manipulated object over three successive angles while starting and/or ending the rotations with predefined fingers positions on the object. The simulation results showed that most trajectories are generated within few seconds. Exploiting adhesion forces enables more feasible trajectories with a lower number of reconfigurations but at the expense of a higher number of nodes (stable grasps) and a longer calculation time. The focus for our future work will be on the fingers collision avoidance and the experimental validation of the proposed method.

Acknowledgment: This work was funded by the EUR EIPHI program (Contract No. ANR-17-EURE-0002), and by HEC Pakistan's HRDI-UESTP program in collaboration with Campus France.

\section{REFERENCES}

[1] R. Ozawa and K. Tahara, "Grasp and dexterous manipulation of multi-fingered robotic hands: a review from a control view point," Advanced Robotics, vol. 31, no. 19-20, pp. 1030-1050, oct 2017. [Online]. Available: https://www.tandfonline.com/doi/full/ 10.1080/01691864.2017.1365011

[2] A. Sahbani, S. El-Khoury, and P. Bidaud, "An overview of 3D object grasp synthesis algorithms," Robotics and Autonomous Systems, vol. 60, no. 3, pp. 326-336, mar 2012. [Online]. Available: http://linkinghub.elsevier.com/retrieve/pii/S0921889011001485

[3] A. M. Okamura, N. Smaby, and M. R. Cutkosky, "An overview of dexterous manipulation," in Proceedings 2000 ICRA. Millennium Conference. IEEE International Conference on Robotics and Automation. Symposia Proceedings (Cat. No. 00CH37065), vol. 1. IEEE, 2000, pp. 255-262.

[4] A. Bicchi and R. Sorrentino, "Dexterous manipulation through rolling," in Proceedings of 1995 IEEE International Conference on Robotics and Automation, vol. 1. IEEE, 1995, pp. 452-457.

[5] D. L. Brock, "Enhancing the dexterity of a robot hand using controlled slip," in Proceedings. 1988 IEEE International Conference on Robotics and Automation. IEEE, 1988, pp. 249-251.

[6] L. Han and J. C. Trinkle, "Dextrous manipulation by rolling and finger gaiting," in Proceedings. 1998 IEEE International Conference on Robotics and Automation (Cat. No. 98CH36146), vol. 1. IEEE, 1998, pp. 730-735.

[7] I. M. Bullock, R. R. Ma, and A. M. Dollar, "A hand-centric classification of human and robot dexterous manipulation," IEEE Transactions on Haptics, vol. 6, no. 2, pp. 129-144, 2013.

[8] O. M. Andrychowicz, B. Baker, M. Chociej, R. Jo, B. Mcgrew, J. Pachocki, A. Petron, M. Plappert, G. Powell, A. Ray, J. Schneider, S. Sidor, J. Tobin, P. Welinder, L. Weng, and W. Zaremba, "Learning dexterous in-hand manipulation," vol. 39, no. 1, pp. 3-20, 2020.

[9] I. M. Bullock, R. R. Ma, and A. M. Dollar, "A hand-centric classification of human and robot dexterous manipulation," IEEE transactions on Haptics, vol. 6, no. 2, pp. 129-144, 2012.

[10] C. Ott, O. Eiberger, W. Friedl, B. Bauml, U. Hillenbrand, C. Borst, A. Albu-Schaffer, B. Brunner, H. Hirschmuller, S. Kielhofer, and et al., A Humanoid Two-Arm System for Dexterous Manipulation, 2006.

[11] Q. Xu, "Design, fabrication, and testing of an mems microgripper with dual-axis force sensor," IEEE Sensors Journal, vol. 15, no. 10, pp. 6017-6026, 2015. 
[12] M. Boudaoud, Y. Haddab, and Y. Le Gorrec, "Modeling and optimal force control of a nonlinear electrostatic microgripper," IEEE/ASME Transactions on mechatronics, vol. 18, no. 3, pp. 1130-1139, 2012.

[13] B. K. Chen, Y. Zhang, D. D. Perovic, and Y. Sun, "Mems microgrippers with thin gripping tips," Journal of Micromechanics and Microengineering, vol. 21, no. 10, p. 105004, 2011.

[14] D. Wang, Q. Yang, and H. Dong, "A monolithic compliant piezoelectric-driven microgripper: Design, modeling, and testing," IEEE/ASmE Transactions on mechatronics, vol. 18, no. 1, pp. 138147, 2011.

[15] X. Sun, W. Chen, S. Fatikow, Y. Tian, R. Zhou, J. Zhang, and M. Mikczinski, "A novel piezo-driven microgripper with a large jaw displacement," Microsystem Technologies, vol. 21, no. 4, pp. 931-942, 2015.

[16] D. Hériban, A. Thiebault, M. Gauthier, and G. Fortier, "Improving rotation behaviour of robotic structures for micro-assembly," in 2008 IEEE International Conference on Automation Science and Engineering. IEEE, 2008, pp. 983-988.

[17] S. Sjövall, Q. Zhou, P. Korhonen, and J. Laitinen, "Automatic dextrous microhandling based on a 6-dof microgripper," Journal of Micromechatronics, vol. 3, no. 3-4, pp. 359-387, 2006.

[18] K. Inoue, T. Tanikawa, and T. Arai, "Micro hand with two rotational fingers and manipulation of small objects by teleoperation," in 2008 International Symposium on Micro-NanoMechatronics and Human Science. IEEE, 2008, pp. 97-102.

[19] H. K. Chu, J. K. Mills, and W. L. Cleghorn, "Automated dual-arm micromanipulation with path planning for micro-object handling," Robotics and Autonomous Systems, vol. 74, pp. 166-174, dec 2015. [Online]. Available: https://linkinghub.elsevier.com/retrieve/pii/ S0921889015001591

[20] T. Chen, Y. Wang, Z. Yang, H. Liu, J. Liu, and L. Sun, "A pzt actuated triple-finger gripper for multi-target micromanipulation," $\mathrm{Mi}$ cromachines, vol. 8, no. 2, p. 33, 2017.

[21] S. Liu, Y.-F. Li, and X.-W. Wang, "A novel dual-probe-based micrograsping system allowing dexterous 3-d orientation adjustment," IEEE Transactions on Automation Science and Engineering, vol. 17, no. 4, pp. 2048-2062, 2020.

[22] J. D. Wason, J. T. Wen, and N. G. Dagalakis, "Dextrous manipulation of a micropart with multiple compliant probes through visual force feedback," in 2011 IEEE International Conference on Robotics and Automation. Ieee, may 2011, pp. 5991-5996. [Online]. Available: http: //ieeexplore.ieee.org/lpdocs/epic03/wrapper.htm?arnumber=5980135

[23] V. Venkatesan and D. J. Cappelleri, "Path Planning and Micromanipulation Using a Learned Model," IEEE Robotics and Automation Letters, vol. 3, no. 4, pp. 3089-3096, oct 2018. [Online]. Available: https://ieeexplore.ieee.org/document/8391723/

[24] R. S. Fearing, "Survey of sticking effects for micro parts handling," in Proceedings 1995 IEEE/RSJ International Conference on Intelligent Robots and Systems. Human Robot Interaction and Cooperative Robots, vol. 2. IEEE, 1995, pp. 212-217.

[25] J. Dejeu, M. Bechelany, L. Philippe, P. Rougeot, J. Michler, and M. Gauthier, "Reducing the adhesion between surfaces using surface structuring with ps latex particle," ACS applied materials \& interfaces, vol. 2, no. 6, pp. 1630-1636, 2010.

[26] J.-A. Seon, R. Dahmouche, and M. Gauthier, "Planning trajectories for dexterous in-hand micro-manipulation using adhesion forces," in 2016 International Conference on Manipulation, Automation and Robotics at Small Scales (MARSS). IEEE, 2016, pp. 1-6.

[27] — "On the contribution of adhesion and friction in planning dexterous in-hand micromanipulation," Journal of Micro-Bio Robotics, vol. 12, no. 1-4, pp. 33-44, 2017.

[28] B. León, A. Morales, and J. Sancho-Bru, From robot to human grasping simulation. Springer, 2014, vol. 19.

[29] J.-A. Seon, R. Dahmouche, and M. Gauthier, "Enhance in-hand dexterous micromanipulation by exploiting adhesion forces," IEEE Transactions on Robotics, vol. 34, no. 1, pp. 113-125, 2017.

[30] L. Euler, "Problema algebraicum ob affectiones prorsus singulares memorabile, novi commentarii academlae scientiarum petropolitanae, 15 (1770) 1771, 75-106," Reprinted in Opera Omnia, I-6, pp. 287-315.

[31] M. Gauthier, S. Alvo, J. Dejeu, B. Tamadazte, P. Rougeot, and S. Régnier, "Analysis and specificities of adhesive forces between microscale and nanoscale," IEEE Transactions on Automation Science and Engineering, vol. 10, no. 3, pp. 562-570, 2013. 